\title{
Effect of melatonin on myocardial oxidative stress induced by experimental obstructive jaundice
}

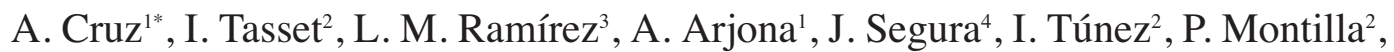 \\ J. Muntané ${ }^{3^{*}}$ and F. J. Padillo ${ }^{1^{*}}$ \\ ${ }^{1}$ Departments of General Surgery, ${ }^{3}$ Research Unit and ${ }^{4}$ Cardiology. Reina Sofía University Hospital. Córdoba, Spain. \\ ${ }^{2}$ Department of Biochemistry and Molecular Biology. School of Medicine. University of Córdoba. Spain. "Centro de \\ Investigación Biomédica en Red de Enfermedades Hepáticas y Digestivas (CIBERehd)
}

\begin{abstract}
Objective: melatonin has been demonstrated to have active antioxidant properties in different tissues during experimental cholestasis. The aim of this research was to study myocardial oxidative stress on obstructive jaundice, and to analyze the effect of melatonin on myocardial oxidative lesions.

Material and methods: we achieved cholestasis by ligature and sectioning of the main bile duct. Melatonin was administered intraperitoneally $(500 \mu \mathrm{g} / \mathrm{kg} /$ day). We measured malondialdehyde (MDA), reduced glutathione (GSH), catalase (CAT), superoxide dismutase (SOD) and glutathione peroxydase (GPx) antioxidant enzyme levels in the heart tissue.

Results: obstructive cholestasis increased MDA and decreased GSH as well as all antioxidant enzymes. Melatonin administration significantly decreased MDA values, and increased GSH and antioxidant enzymes on the icteric animal myocardium.

Conclusions: melatonin treatment prevents oxidative stress in the cardiac tissue as induced by experimental cholestasis.
\end{abstract}

Key words: Antioxidants. Cholestasis. Cardiac dysfunction. Melatonin. Oxidative stress.

Cruz A, Tasset I, Ramírez LM, Arjona A, Segura J, Túnez I, Montilla P, Muntané J, Padillo FJ. Effect of melatonin on myocardial oxidative stress induced by experimental obstructive jaundice. Rev Esp Enferm Dig 2009; 101: 460-463.

Received: 03-03-09.

Accepted: 12-03-09.

Correspondence: Francisco J. Padillo. Unidad de Cirugía Pancreática y Programa de Trasplante Pancreático. Departamento de Cirugía. Hospital Universitario Reina Sofía. Avda. Menéndez Pidal, s/n. 14004 Córdoba, Spain.e-mail: javierpadiruiz@hotmail.com

\section{INTRODUCTION}

Biliary obstruction has an important effect both on the liver and extrahepatic organs (1). Obstructive jaundice (OJ) increases oxidative stress, characterized by a rise of systemic malondialdehyde (MDA) and a decrease in cellular antioxidant defenses, such as reduced glutathione (GSH) and antioxidant enzymes (2). The rise of different parameters of oxidative stress during experimental OJ has been shown in serum (2), hepatic (2) and renal (3) parenchyma, and on the central nervous system, specifically on the hypothalamus, bulb and cerebral cortex (4). Oxidative stress has been related to serious complications in liver (5-7) and renal dysfunction (3). OJ has been shown to induce hypotension and fluid depletion (8). Moreover, it has also been suggested that biliary obstruction causes depression mainly in myocardial contractility of the left ventricle (9). Melatonin is a hormone with great antioxidant capacity (10). Its beneficial effects against oxidative stress have also been demonstrated in liver cirrhosis (11) and acute pancreatitis (12). The aims of our study were firstly to measure oxidative stress in the myocardium tissue after biliary obstruction, and secondly to demonstrate the antioxidant properties of melatonin in myocardial tissue during obstructive cholestasis.

\section{METHODS}

\section{Animals}

Male Wistar rats (250-330 g) were subjected to controlled conditions of temperature (about $22-24{ }^{\circ} \mathrm{C}$ ) and illumination (12-h light: 12-h dark cycle), and were provided with food (Purina ${ }^{\oplus}$, Barcelona, Spain) and water $a d$ libitum. Animals were treated according to institutional guidelines, and the study was approved by the Research Committee of the Reina Sofía University Hospital. 


\section{Experimental design}

Animals ( $\mathrm{n}=21,7$ in each group) were distributed in each of the following groups: sham operated (SO), OJ, and OJ plus melatonin (OJ + MEL). All the surgical procedures were done in animals anesthetized with ketamine $(60 \mathrm{mg} / \mathrm{kg}$ i.p.) and midazolam (4 mg/kg i.p.). Cephazoline (17 mg/kg i.m.) was used as antibiotic prophylaxis. SO animals were submitted to laparotomy and abdominal closure without bile duct intervention. The procedure for OJ was started by a midline ventral incision with exposure of the extra-hepatic bile duct. A double ligature with silk suture was done and the bile duct was sectioned. A two-layer running suture was used for abdominal closure with polyglycolic acid and silk. Melatonin (500 $\mu \mathrm{g} / \mathrm{kg} /$ day i.p.; Sigma-Aldrich, St Louis, $\mathrm{MO}$ ) was diluted in $5 \%$ ethanol in $0.9 \% \mathrm{NaCl}$. The administration of the solvent did not change the parameters (data not shown). Melatonin was injected daily at $10 \mathrm{~h}$ starting the day before the bile duct ligature was made, and until the day preceding the sacrifice. The animals were sacrificed under anesthesia 7 days after OJ. Blood was collected from the abdominal aorta and serum was frozen at $-80{ }^{\circ} \mathrm{C}$ until the measurement of alkaline phosphatase (AP) and total bilirubin. The heart was dissected, aliquot and immediately frozen at $-80{ }^{\circ} \mathrm{C}$ until the measurements could be taken. Tissue was homogenized in ice-cold phosphate-EDTA buffer $(10 \mathrm{mM}$ phosphate buffer [pH 7.2], containing $1 \mathrm{mM}$ EDTA and $1 \mathrm{mM}$ BHA diluted in $0.15 \%$ ethanol) using a glassglass Potter-Elvehjem homogenizer.

\section{Biochemical assays}

Total bilirubin and AP were measured in serum using an Axon analyzer (Bayer Diagnostics, Tarrytown, NY). MDA was determined in tissue according to the procedure described in the assay (LPO-586, Bioxytec, Portland, OR). A GSH-400 assay commercial kit (Bioxytec, Portland, OR) was used for measuring GSH in tissue. Catalase (CAT) and superoxide dismutase (SOD) activities were measured according to Aebi (13) and Sun (14), respectively. Glutathione peroxidase (GPx) was measured according to Flohe and Gunzler (15).

\section{Statistical analysis}

The results are expressed as means with their corresponding standard errors. Homogeneity of variance was assessed with Levene's test. Comparisons were made using the two-tailed, unpaired Student's t test and ANOVA test. Statistical significance was set at $\mathrm{p}<0.05$.

\section{RESULTS}

The biliary obstruction model caused acute cholestasis in OJ animals, characterized by a significant increase in both total bilirubin and AP in comparison with SO animals (Table I). Regarding this effect, animals which underwent bile-duct obstruction had a significant increase in the MDA oxidative stress marker in the myocardium (Fig. 1). Moreover, a positive correlation between serum AP and MDA concentration has been shown in myocardial tissue (Fig. 2). In addition to increased lipoperoxides, the concentration of antioxidant defenses was drastically reduced in the myocardium of OJ animals. In this respect, OJ induced a significant decrease in GSH (Fig. 3) and in antioxidant enzymes SOD, CAT and GPx (Fig.4).

On the other hand, melatonin administration to icteric animals determined a significant restoration of oxidative stress markers in the myocardium. Melatonin decreased MDA concentration and increased GSH (Figs. 1 and 3), and likewise recovered the activity of all the enzymatic antioxidants studied (Fig. 4).

\section{DISCUSSION}

The study of the involvement of oxidative stress during OJ has identified several potential agents that exert a beneficial effect against tissue injury induced by biliary obstruction. Oxidative stress by OJ is characterized by a rise of lipoperoxides and a reduction of cellular antioxidant status. A rise in MDA in the liver parenchyma and serum has been observed in animals with biliary obstruction (2). The reduction in blood antioxidant concentration may have a relevant repercussion on different tissues during OJ. In addition, free radicals may originate in extrahepatic organs or in organs not related to the biliary tree during OJ. We have proven that there is increased oxidative stress in the kidney (3) and central nervous system (4) that

Table I. Cholestatic markers in the serum of rats with obstructive jaundice

\begin{tabular}{lccccc}
\hline & SO & OJ & $p$ & OJ-MEL & $p^{*}$ \\
\hline Total bilirubin $(\mathrm{mg} / \mathrm{dL})$ & $0.12 \pm 0.030$ & $9.5 \pm 0.80$ & $<0.0001$ & $7.7 \pm 0.10$ & $<0.05$ \\
AP $(\mathrm{U} / \mathrm{L})$ & $86.5 \pm 21.79$ & $378 \pm 26.0$ & $<0.0001$ & $354 \pm 13.2$ & n.s. \\
\hline
\end{tabular}

*In relation to the OJ group. SO: Sham operated; OJ: Obstructive jaundice group; OJ-MEL: Animals treated with melatonin after biliary obstruction. 


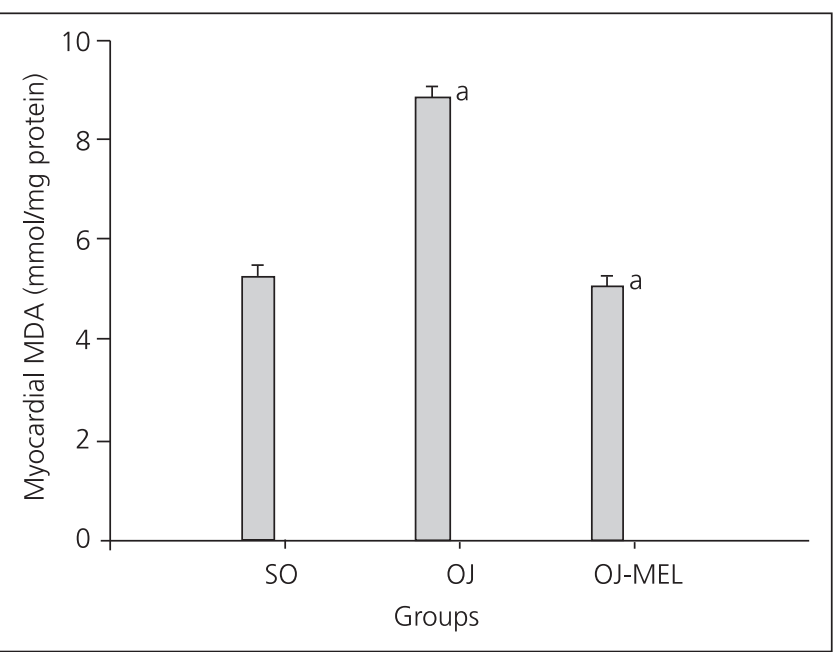

Fig. 1. Reduction of malondialdehyde (MDA) content by melatonin in cardiac tissue from obstructed animals. Obstructive jaundice (OJ) was induced in control animals (SO) by bile duct ligation. The OJ-MEL group was submitted to OJ and received melatonin $(500 \mu \mathrm{g} / \mathrm{kg}$ ) daily until their sacrifice 7 days after the surgical intervention. MDA was determined in myocardial tissue following the procedure described in "Animals" (Methods). Statistical significance was set at $p \leq 0.0001$; ${ }^{a}$ significantly different from the SO group.

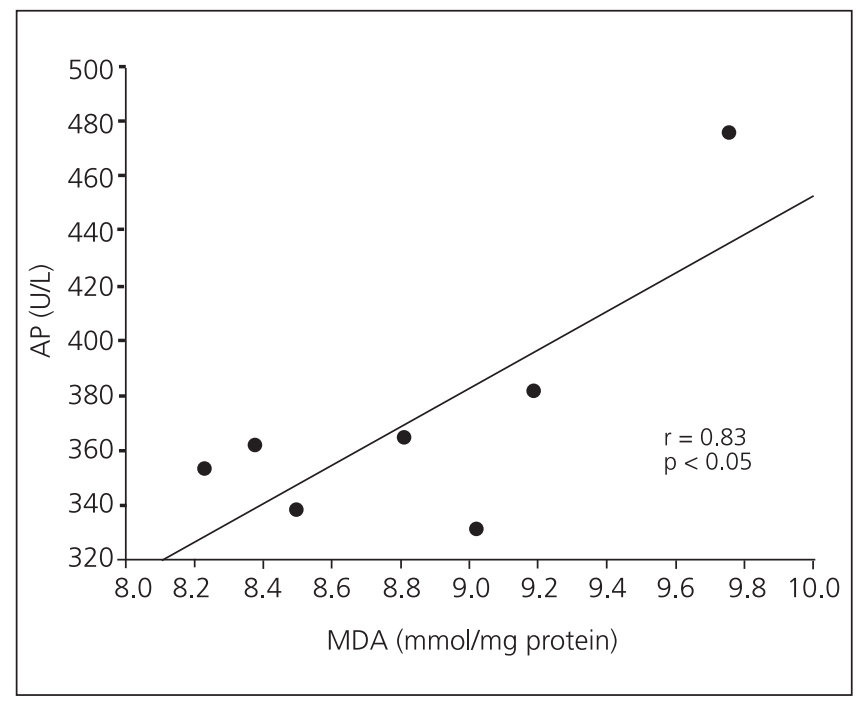

Fig. 2. Correlation analysis between alkaline phosphatase (AP) and malondialdehyde (MDA) in animals submitted to bile duct ligation (OJ).

may explain various complications during cholestasis. On the lines of the impact of biliary obstruction on extrahepatic organs, this study proves for the first time an increase in MDA and a decrease in GSH and antioxidant enzymes in the myocardium from OJ rats. It has been recognized that bile-duct obstruction has a detrimental effect on cardiovascular physiology (16). A more recent study has shown a reverse correlation between the left ventricle systolic work and bilirubin in obstructive jaundice patients, suggesting that bile-duct obstruction has a depressor effect on myocardial contractility, mainly in the left ventri-

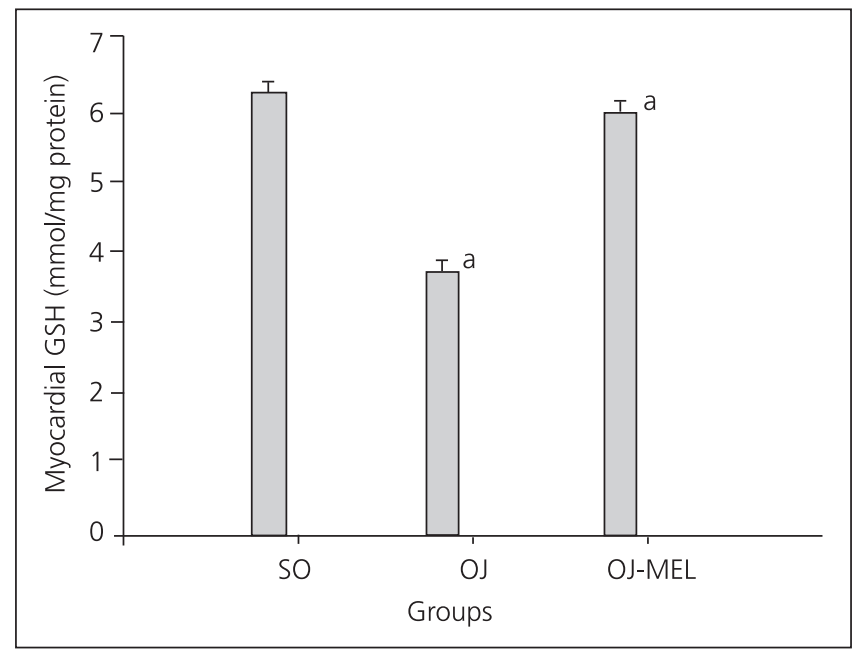

Fig. 3. Recovery of reduced glutathione (GSH) content by melatonin in the myocardium tissue from obstructed animals. Obstructive jaundice (OJ) was induced in control animals (SO) by bile duct ligation. The OJtreated animals (OJ-MEL) received melatonin $(500 \mu \mathrm{g} / \mathrm{kg}$ ) daily until their sacrifice 7 days after the surgical intervention. GSH was determined in myocardium tissue following the procedure described in "Animals" (Methods). Statistical significance was set at $p \leq 0.0001$; ${ }^{a}$ significantly different from the SO group.

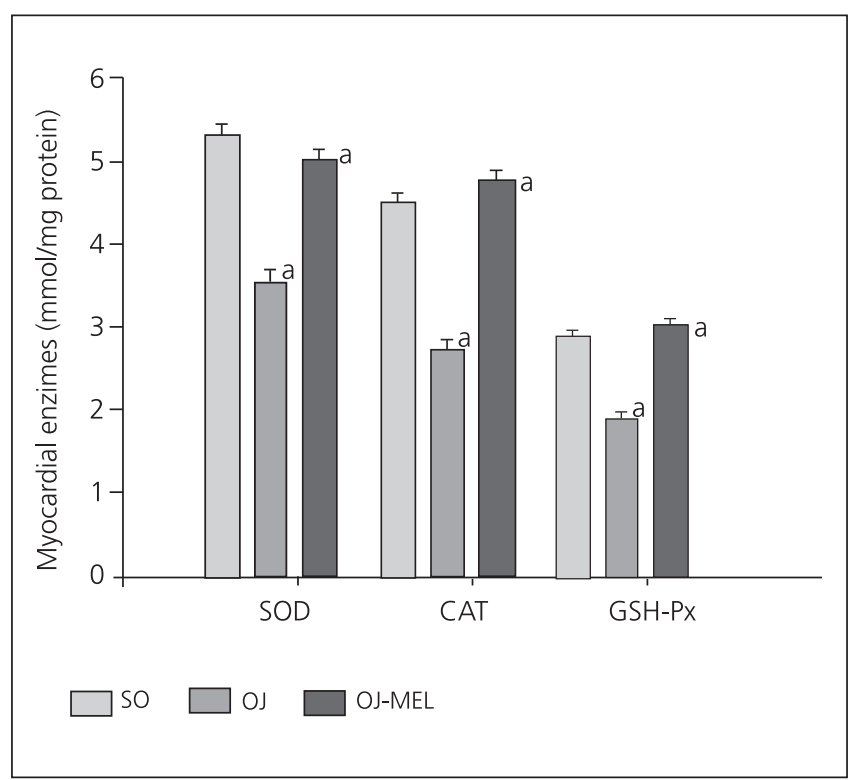

Fig. 4. Recovery of antioxidant status by melatonin in cardiac tissue from obstructed animals. Obstructive jaundice (OJ) was induced in control animals (SO) by bile duct ligation. The OJ-MEL group was submitted to OJ and received melatonin $(500 \mu \mathrm{g} / \mathrm{kg}$ ) daily until their sacrifice 7 days after the surgical intervention. Superoxide dismutase (SOD), catalase (CAT) and glutathione peroxidase (GSH-Px) were measured in myocardium tissue following the procedure described in "Animals" (Methods). Statistical significance was set at $p \leq 0.0001$; ${ }^{a}$ significantly different from the SO group.

cle (8). This fact could help explain the pathophysiology of shock in patients suffering from severe cholangitis, and could be important for icteric patients with previous heart disease. In addition to that, in the above-mentioned study, 
biliary drainage allowed a normalization of hemodynamic parameters (8). The existence of a higher redox state in patients suffering from cardiac insufficiency has been proven, this being correlated with functional parameters; so there is a reverse connection between MDA figures and ventricular ejection fraction (17). Although we have not evaluated hemodynamic dysfunction in our study, we have clearly demonstrated an increase in myocardial oxidative stress, which could be involved in myocardial dysfunction in OJ rats. Moreover, a positive correlation between AP and MDA in the myocardium has been proven, which suggests an increase in myocardium oxidative damage when a worsening of cholestasis occurs. Although surgical procedures for cholestasis, such as biliary drainage, are suitable for the treatment of OJ, we have successfully tested antioxidant therapies to reduce the oxidative damage associated with OJ. Melatonin has demonstrated hepatoprotective properties on experimental cholestasis (5) and drastically improves oxidative stress parameters in the renal parenchyma (3). The discovery of melatonin as a direct scavenger of free radicals and as an indirect antioxidant stimulating antioxidant enzymes has raised interest in its potential cardioprotective properties (18). Our study suggests that the cardioprotection of melatonin was related to a reduction of MDA and a restoration of the antioxidant status in the myocardium. Melatonin had beneficial effects on other myocardial dysfunctions such as ischemia reperfusion (19), reducing areas with heart congestion (20). On the basis of experimental results, we suggest that melatonin could have a protective effect on myocardial dysfunction generated in OJ that may be relevant in order to decrease the systemic complications of these patients. Melatonin has demonstrated antihypertensive properties (21) that would have a beneficial impact on ventricular function. In addition, melatonin has pharmacological advantages such as oral administration, excellent tissue distribution both in fat-soluble and watersoluble environments, and its capacity to be highly concentrated in every section of the cell, especially in mitochondria, which is very important for myocardial cells (18).

In conclusion, myocardial biochemical oxidative dysfunction induced by biliary obstruction may be counteracted upon by melatonin. However, we should carry out new clinical trials to prove the benefits of antioxidant therapy with melatonin, and to help decrease mortality rates among patients with obstructive cholestasis.

\section{ACKNOWLEDGEMENTS}

This study has been supported by the Instituto de Salud Carlos III (FIS 02/0181, FIS 05/0703) and the Con- sejería de Salud (SAS 50/03). CIBERehd is funded by the Instituto de Salud Carlos III.

\section{REFERENCES}

1. Pérez-Fernández T, López-Serrano P, Tomás E, Gutiérrez ML, Lledó JL, Cacho G, et al. Diagnostic and therapeutic approach to cholestatic liver disease. Rev Esp Enferm Dig 2004; 96: 60-73.

2. Montilla P, Cruz A, Padillo FJ, Tunez I, Gascon F, Muñoz MC, et al. Melatonin versus vitamin $\mathrm{E}$ as protective treatment against oxidative stress after extrahepatic bile duct ligation in rats. J Pineal Res 2001; 31: 138-44.

3. Cruz A, Padillo FJ, Túnez I, Muñoz C, Granados J, Pera-Madrazo C, et al. Melatonin protects against renal oxidative stress after obstructive jaundice in rats. Eur J Pharmacol 2001; 425: 135-9.

4. Cruz A, Túnez I, Martínez R, Muñoz-Castañeda JR, Ramírez LM, Recio M, et al. Melatonin prevents brain oxidative stress induced by obstructive jaundice in rats. J Neurosci Res 2007; 85: 3652-6.

5. Padillo FJ, Cruz A, Navarrete C, Bujalance I, Briceño J, Gallardo JI, et al. Melatonin prevents oxidative stress and hepatocyte cell death induced by experimental cholestasis. Free Radic Res 2004; 38: 697-704.

6. Conde de la Rosa L, Moshage H, Nieto N. Hepatocyte oxidant stress and alcoholic liver disease. Rev Esp Enferm Dig 2008; 100: 156-63.

7. Muntané J, González R, Ranchal I, Collado JA, López-Sánchez L, Herencia C, et al. Mechanisms of liver injury. Rev Esp Enferm Dig 2007; 99: 405-10.

8. Padillo FJ, Rodríguez M, Gallardo JM, Andicoberry B, Naranjo A, Miño G, et al. Preoperative assessment of body fluid disturbances in patients with obstructive jaundice. Word J Surg 1999; 23: 681-7.

9. Padillo J, Puente J, Gómez M, Dios F, Naranjo A, Vallejo JA, et al. Improved cardiac function in patients with obstructive jaundice after internal biliary drainage. Hemodynamic and hormonal assessment. Ann Surg 2001; 234: 652-6.

10. Reiter RJ, Tan Dx, Terron MP, Flores LJ, Czarnocki Z. Melatonin and its metabolites: new findings regarding their production and their radical scavenging actions. Acta Biochim Pol 2007; 54: 1-9.

11. Cruz A, Padillo FJ, Torres E, Navarrete CM, Muñoz-Castañeda JR, Caballero FJ, et al. Melatonin prevents experimental liver cirrhosis induced by thioacetamide in rats. J Pineal Res 2005; 39: 143-50.

12. Muñoz-Casares FC, Padillo FJ, Briceño J, Collado JA, Muñoz-Castañeda JR, Ortega R, et al. Melatonin reduces apoptosis and necrosis induced by ischemia/reperfusion injury of the pancreas. J Pineal Res 2006; 40: 195-203.

13. Aebi H. Catalase in vitro. Methods Enzymol 1984; 105: 121-6.

14. Sun Y, Oberley LW, Li Y. A simple method for clinical assay of superoxide dismutase. Clin Chem 1988; 34: 479-500.

15. Flohe L, Gunzler WA. Assays of glutathione peroxidase. Methods Enzymol 1984; 105: 114-21.

16. Better OS, Bomzon A. Effects of jaundice on the renal and cardiovascular systems. In: Epstein M, editor. The kidney in liver disease. $3^{\text {rd }}$ ed. Baltimore: Willians \& Wilkins; 1988. p. 508-34.

17. Sam F, Kerstetter DL, Pimental DR, Mulukutla S, Tabaee A, Bristow $\mathrm{MR}$, et al. Increased reactive oxygen species production and functional alterations in antioxidant enzymes in human mailing myocardium. Congest Heart Fail 2005; 11: 2130-44.

18. Tengattini S, Reiter RJ, Tan DX, Terron P, Rodella LF, Rezani R. Cardiovascular diseases: protective effects of melatonin. J Pineal Res 2008; 44: 16-25.

19. Kaneko S, Okumura K, Numaguchi Y, Matsui H, Murase K, Mokuno $\mathrm{S}$, et al. Melatonin scavenges hydroxyl radical and protects isolated rat hearts from ischemic reperfusion injury. Life Sci 2000; 67: 101-12.

20. Lagneux C, Joyeux M, Demente P, Ribuot C, Godin-Ribuot D. Protective effects of melatonin against ischemia-reperfusion injury in the isolated rat heart. Life Sci 2000; 66: 503-9.

21. Simko P, Paulis L. Melatonin as a potential antihypertensive treatment. J Pineal Res 2007; 42: 319-22. 